\title{
A System of Nonlinear Operator Equations for a Mixed Family of Fuzzy and Crisp Operators in Probabilistic Normed Spaces
}

\author{
Yeol Je Cho, ${ }^{1}$ Heng-you Lan, ${ }^{2}$ and Nan-jing Huang ${ }^{3}$ \\ ${ }^{1}$ Department of Mathematics Education and the RINS, Gyeongsang National University, \\ Chinju 660-701, South Korea \\ ${ }^{2}$ Department of Mathematics, Sichuan University of Science E Engineering, Zigong, \\ Sichuan 643000, China \\ ${ }^{3}$ Department of Mathematics, Sichuan University, Chengdu, Sichuan 610064, China
}

Correspondence should be addressed to Heng-you Lan, hengyoulan@163.com

Received 8 January 2010; Accepted 23 March 2010

Academic Editor: Yuming Xing

Copyright (C) 2010 Yeol Je Cho et al. This is an open access article distributed under the Creative Commons Attribution License, which permits unrestricted use, distribution, and reproduction in any medium, provided the original work is properly cited.

By using a random version of the theory of contractor introduced by Altman, we introduce and study a system of nonlinear operator equations for a mixed family of fuzzy and crisp operators in probabilistic normed spaces. We construct some new iterative algorithms for solving this kind of nonlinear operator equations. We also prove some new existence theorems of solutions of a new system of nonlinear operator equations for a mixed family of fuzzy and crisp operators and some new convergence results of sequences generated by iterative algorithms under joint orbitally complete conditions.

\section{Introduction}

Altman [1, 2] introduced the theory of contractor and contractor direction, which has a very strong significant for the study of existence and uniqueness for solving nonlinear operator equations in Banach spaces. The theory of contractor offers a unified approach to a very large class of iterative methods including the most important ones. Chang [3] introduced the concept of probabilistic contractor and studied the existence and uniqueness of solution for nonlinear operator equations with probabilistic contractor in Menger PN-spaces. By using the theory of countable extension of $t$-norms [4-6] and the results from $[7,8]$, many results for the more general classes of $t$-norms have been proved (see [9] and the references therein).

On the other hand, since then, several kinds of variational inequalities, variational inclusions, complementarity problems, and nonlinear equations with fuzzy mappings were 
introduced and studied by many authors (see, e.g., [8-15]). Sharma et al. [15] considered two nonfuzzy mappings and a sequence of fuzzy mappings to define a hybrid $D$-compatible condition. They also showed the existence of common fixed points under such condition, where the range of the one of the two nonfuzzy mappings is joint orbitally complete. Furthermore, Cho et al. [10] introduced the concept of probabilistic contractor couple in probabilistic normed spaces and discuss the solution for nonlinear equations of fuzzy mappings and the convergence of sequences generated by the algorithms in Menger probabilistic normed spaces. Very recently, Hadžić and Pap [16] introduced some new classes of probabilistic contractions in probabilistic metric spaces. They also obtained a new fixed point theorem for the $\psi$-probabilistic contraction and gave some applications to random operators.

Motivated and inspired by the above works, in this paper, by using a random version of the theory of contractor introduced by Altman, we introduce and study a system of nonlinear operator equations for a mixed family of fuzzy and crisp operators in probabilistic normed spaces. We construct some new iterative algorithms for solving this kind of nonlinear operator equations. We also prove some new existence theorems of solution for the system of nonlinear operator equations for a mixed family of fuzzy and crisp operators and new convergence results of sequences generated by the iterative algorithms under joint orbitally complete condition. The results presented in this paper improve and generalize corresponding results of $[9,15-17]$.

\section{Preliminaries}

Let $\Delta^{+}$be the set of all distribution functions $F$ such that $F(0)=0$ ( $F$ is a nondecreasing, left continuous mapping from $\mathbb{R}$ into $[0,1]$ such that $\left.\sup _{x \in \mathbb{R}} F(x)=1\right)$. The special distribution function $H$ is defined by

$$
H(t)= \begin{cases}1, & t>0 \\ 0, & t \leq 0\end{cases}
$$

The ordered pair $(S, \mathcal{F})$ is said to be a probabilistic metric space if $S$ is a nonempty set and $\mathcal{F}: S \times S \rightarrow \Delta^{+}\left(\mathcal{F}(p, q)\right.$ is written by $F_{p, q}$ for all $\left.(p, q) \in S \times S\right)$ satisfying the following conditions:

(i) $F_{u, v}(x)=1$ for all $x>0 \Leftrightarrow u=v$ for all $u, v \in S$;

(ii) $F_{u, v}=F_{v, u}$ for all $u, v \in S$;

(iii) $F_{u, v}(x)=1$ and $F_{v, w}(y)=1 \Rightarrow F_{u, w}(x+y)=1$ for all $u, v, w \in S$ and $x, y \in \mathbb{R}^{+}$.

A Menger space (see [18]) is a triple $(S, \mathcal{F}, T)$, where $(S, \mathscr{F})$ is a probabilistic metric space, $T$ is a triangular norm (abbreviated $t$-norm), and the following inequality holds:

$$
F_{u, v}(x+y) \geq T\left(F_{u, w}(x), F_{w, v}(y)\right), \quad \forall u, v, w \in S, x, y>0
$$


Recall that a mapping $T:[0,1] \times[0,1] \rightarrow[0,1]$ is a triangular norm (shortly, a $t$-norm) if the following conditions are satisfied:

(i) $T(a, 1)=a$ for all $a \in[0,1]$;

(ii) $T(a, b)=T(b, a)$ for all $a, b \in[0,1]$;

(iii) if $a \geq b$ and $c \geq d$, then $T(a, c) \geq T(b, d)$ for all $a, b, c, d \in[0,1]$;

(iv) $T(a, T(b, c))=T(T(a, b), c)$ for all $a, b, c \in[0,1]$.

Example 2.1. The following are the four basic examples.

(1) The minimum $t$-norm $T_{M}$ is defined by

$$
T_{M}(x, y)=\min (x, y)
$$

(2) The product $t$-norm $T_{P}$ is defined by

$$
T_{P}(x, y)=x \cdot y
$$

(3) The Lukasiewicz $t$-norm $T_{L}$ is defined by

$$
T_{L}(x, y)=\max (x+y-1,0)
$$

(4) The weakest $t$-norm, the drastic product $T_{D}$, is defined by

$$
T_{D}(x, y)= \begin{cases}\min (x, y), & \text { if } \max (x, y)=1 \\ 0, & \text { otherwise }\end{cases}
$$

The $(\epsilon, \lambda)$-topology in $S$ is introduced by the family of neighbourhoods $u=$ $\left\{U_{v}(\epsilon, \lambda)\right\}_{(v, \epsilon, \lambda) \in S \times \mathbb{R}_{+} \times(0,1)}$, where

$$
U_{v}(\epsilon, \lambda)=\left\{u ; F_{u, v}(\epsilon)>1-\lambda\right\} .
$$

If a $t$-norm $T$ is such that $\sup _{x<1} T(x, x)=1$, then $S$ is a metrizable topological space under the $(\epsilon, \lambda)$-topology.

Let $X$ be a vector space over the real or complex number field $\mathcal{K}, \mathcal{F}: X \rightarrow \Delta^{+}$, and $T$ a $t$-norm. The ordered triple $(X, \mathcal{F}, T)$ is a Menger probabilistic normed space (briefly, a Menger PN-space) if and only if the following conditions are satisfied, where $\mathscr{F}(x)=F_{x}$ for all $x \in S$ :

(i) $F_{x}(0)=0$ for all $x \in S$ and $F_{x}=H \Leftrightarrow x=\theta(\theta$ is a neutral element for + in $X)$;

(ii) $F_{\lambda x}(t)=F_{x}(t /|\lambda|)$ for all $x \in X$ and $\lambda \in \mathcal{K}(\lambda \neq 0)$;

(iii) $F_{x+y}\left(t_{1}+t_{2}\right) \geq T\left(F_{x}\left(t_{1}\right), F_{y}\left(t_{2}\right)\right)$ for all $x, y \in X$ and $t_{1}, t_{2}>0$. 
If $(X, \mathcal{F}, T)$ is a Menger PN-space and, for all $x, y \in X, \bar{\mp}: X \times X \rightarrow \Delta^{+}$is defined by

$$
\overline{\mathcal{F}}(x, y)=F_{x-y},
$$

then $(X, \bar{\mp}, T)$ is a Menger space.

The following definition can be found in [7].

Definition 2.2 (see [7]). (X, $\mathscr{F}, T)$ is called a non-Archimedean Menger PN-space (briefly, an N.A. Menger PN-space) if $(X, \mathcal{F}, T)$ is a Menger PN-space satisfying the following condition:

$$
F_{x+y}\left(\max \left\{t_{1}, t_{2}\right\}\right) \geq T\left(F_{x}\left(t_{1}\right), F_{y}\left(t_{2}\right)\right), \quad \forall x, y \in X, t_{1}, t_{2} \geq 0
$$

If $(X, \mathcal{F}, T)$ is a Menger PN-space and $T$ is a $t$-norm which satisfies the condition:

$$
\sup _{a<1} T(a, a)=1
$$

then $(X, \mathcal{F}, T)$ is a Hausdorff topological vector space in the topology $\tau$ induced by the base of neighborhoods of $\theta$

$$
\{U(\varepsilon, \lambda) \mid \varepsilon>0, \lambda \in(0,1]\},
$$

where $U(\varepsilon, \lambda)=\left\{x \in X: F_{x}(\varepsilon)>1-\lambda\right\}$.

Example 2.3 (see [9]). It is easy to see that an ultra-metric space $(M, d)$ belongs to the class of N.A. Menger PN-spaces, where $M \neq \emptyset$ and $d: M \times M \rightarrow[0, \infty)$ satisfying the following conditions:

(i) $d(x, y)=0 \Leftrightarrow x=y$ for all $x, y \in M$;

(ii) $d(x, y)=d(y, x)$ for all $x, y \in M$;

(iii) $d(x, z) \leq \max \{(x, y), d(y, z)\}$ for all $x, y, z \in M$.

A fuzzy set $A$ in $X$ is a function from $X$ into [0,1]. If $x \in X$, then the function value $A(x)$ is called the grade of membership of $x$ in $A$. The $\alpha$-level set of $A$, denoted by $A_{\alpha}$, is defined by

$$
A_{\alpha}=\{x: A(x) \geq \alpha\}, \quad \forall \alpha \in(0,1]
$$

Let $W(X)$ denote the collection of all fuzzy sets $A$ in $X$ such that $A_{\alpha}$ is compact and convex for all $\alpha \in(0,1]$ and $\sup _{x \in X} A(x)=1$. For any $A, B \in W(X), A \subset B$ means $A(x) \leq B(x)$ for all $x \in X$.

Let $M$ be an arbitrary set and $X$ any linear metric space. A function $\widetilde{S}: M \rightarrow W(X)$ is called fuzzy operator. Now, we define an orbit for mixed operators $\left(\widetilde{S}_{n}, f, g\right)$ and a joint orbitally complete space as follows. 
Definition 2.4 (see [15]). Let $f, g$ be two operators from $X$ into itself and $\left\{\widetilde{S}_{n}\right\}_{n=1}^{\infty}$ a sequence of fuzzy operators from $X$ into $W(X)$. If, for some $x_{0} \in X$, there exist sequences $\left\{x_{n}\right\}$ and $\left\{y_{n}\right\}$ in $X$ such that

$$
\left\{\begin{array}{l}
\left\{y_{2 n+1}\right\}=\left\{f\left(x_{2 n+1}\right)\right\} \subset \widetilde{S}_{2 n+1}\left(x_{2 n}\right), \\
\left\{y_{2 n+2}\right\}=\left\{g\left(x_{2 n+2}\right)\right\} \subset \widetilde{S}_{2 n+2}\left(x_{2 n+1}\right), \quad \forall n \geq 0,
\end{array}\right.
$$

then $\vartheta\left(\widetilde{S}_{n}, f, g, x_{0}\right)=\left\{y_{n}: n \geq 1\right\}$ is called an orbit for the mixed operators $\left(\widetilde{S}_{n}, f, g\right)$.

Definition 2.5 (see [15]). $X$ is called $x_{0}$-joint orbitally complete if every Cauchy sequence of each orbit at $x_{0}$ is convergent in $X$.

Remark 2.6 (see [15]). Clearly, if $X$ is an any complete space and $x_{0} \in X$, then $X$ is $x_{0}$-joint orbitally complete, while the converse is not necessarily true.

\section{Some Countable $t$-Norms}

Let $T$ be a $t$-norm and, for each $n \geq 1$, and a mapping $T_{n}:[0,1] \rightarrow[0,1]$ let defined in the following way:

$$
T_{1}(x)=T(x, x), \quad T_{n+1}(x)=T\left(T_{n}(x), x\right), \quad \forall n \geq 1, x \in[0,1] .
$$

A $t$-norm $T$ is of $H$-type if the family $\left\{T_{n}(x)\right\}_{n \geq 1}$ is equicontinuous at $x=1$ (see [19]).

Each $t$-norm $T$ can be extended (by the associativity) in a unique way to the $n$-ary operation taking the values $T\left(x_{1}, x_{2}, \ldots, x_{n}\right)=\mathbf{T}_{i=1}^{n} x_{i}$ for any $\left(x_{1}, \ldots, x_{n}\right) \in[0,1]^{n}$, which is defined by

$$
\mathbf{T}_{i=1}^{0} x_{i}=1, \quad \mathbf{T}_{i=1}^{n} x_{i}=T\left(\mathbf{T}_{i=1}^{n-1} x_{i}, x_{n}\right) .
$$

A $t$-norm $T$ can be extended to countable infinitely operations taking the value

$$
\mathbf{T}_{i=1}^{\infty} x_{i}=\lim _{n \rightarrow \infty} \mathbf{T}_{i=1}^{n} x_{i}
$$

for any sequence $\left\{x_{n}\right\}_{n \geq 1}$ in $[0,1]$. Also, the sequence $\left\{\mathbf{T}_{i=1}^{n} x_{i}\right\}_{n \geq 1}$ is nonincreasing and bounded from below and hence the limit $\mathrm{T}_{i=1}^{\infty} x_{i}$ exists.

By (3.3) and fixed point theory in the book by Hadžić and Pap [4], it is interested to investigate the classes of $t$-norms $T$ and sequences $\left\{x_{n}\right\}$ in the interval $[0,1]$ such that $\lim _{n \rightarrow \infty} x_{n}=1$ and

$$
\begin{aligned}
\lim _{n \rightarrow \infty} \mathbf{T}_{i=n}^{\infty} x_{i} & \triangleq \lim _{n \rightarrow \infty} \lim _{m \rightarrow \infty} \mathbf{T}_{i=n}^{m} x_{i} \\
& =\lim _{n \rightarrow \infty} \lim _{m \rightarrow \infty} \mathbf{T}_{i=1}^{m-n+1} x_{i+n-1} \\
& =\lim _{n \rightarrow \infty} \mathbf{T}_{i=n}^{\infty} x_{i+n-1} \\
& =\lim _{n \rightarrow \infty} \mathbf{T}_{i=1}^{\infty} x_{n+i-1}=1 .
\end{aligned}
$$


It is obvious that

$$
\lim _{n \rightarrow \infty} \mathbf{T}_{i=n}^{\infty} x_{i}=1 \Longleftrightarrow \sum_{i=1}^{\infty}\left(1-x_{i}\right)<\infty
$$

for $T=T_{L}$ and $T=T_{P}$.

The important classes of $t$-norms are given in the following example.

Example 3.1 (see [16]). (1) The Dombi family of $t$-norms $\left(T_{\lambda}^{D}\right)_{\lambda \in[0, \infty]}$ is defined by

$$
T_{\lambda}^{D}(x, y)= \begin{cases}T_{D}(x, y), & \lambda=0, \\ T_{M}(x, y), & \lambda=\infty, \\ \frac{1}{1+\left(((1-x) / x)^{\lambda}+((1-y) / y)^{\lambda}\right)^{1 / \lambda}}, & \lambda \in(0, \infty) .\end{cases}
$$

(2) The Aczél-Alsina family of $t$-norms $\left(T_{\lambda}^{\mathrm{AA}}\right)_{\lambda \in[0, \infty]}$ is defined by

$$
T_{\lambda}^{\mathrm{AA}}(x, y)= \begin{cases}T_{D}(x, y), & \lambda=0, \\ T_{M}(x, y), & \lambda=\infty, \\ e^{-\left((-\log x)^{\lambda}+(-\log y)^{\lambda}\right)^{1 / \lambda},} & \lambda \in(0, \infty) .\end{cases}
$$

(3) The family $\left(T_{\lambda}^{S W}\right)_{\lambda \in[-1, \infty]}$ of Sugeno-Weber $t$-norms is given by

$$
T_{\lambda}^{\mathrm{SW}}(x, y)= \begin{cases}T_{D}(x, y), & \lambda=-1, \\ T_{P}(x, y), & \lambda=\infty, \\ \max \left(0, \frac{x+y-1+\lambda x y}{1+\lambda}\right), & \lambda \in(-1, \infty) .\end{cases}
$$

(4) The Schweizer-Sklar family of $t$-norms $\left(T_{\lambda}^{S S}\right)_{\lambda \in[-\infty, \infty]}$ is defined by

$$
T_{\lambda}^{S S}(x, y)= \begin{cases}T_{M}(x, y), & \lambda=-\infty, \\ T_{P}(x, y), & \lambda=0, \\ \left(\max \left(x^{\curlywedge}+y^{\curlywedge}-1,0\right)\right)^{1 / \lambda}, & \lambda \in(-\infty, 0) \cup(0, \infty), \\ T_{D}(x, y), & \lambda=\infty .\end{cases}
$$

The condition $T \geq T_{L}$ is fulfilled by the families $\left(T_{\lambda}^{S S}\right)_{\lambda \in(-\infty, 1)},\left(T_{\lambda}^{S W}\right)_{\lambda \in[0, \infty]}$.

There exists a member of the family $\left(T_{\lambda}^{D}\right)_{\lambda \in(0, \infty)}$ which is incomparable with $T_{L}$ and there exists a member of the family $\left(T_{\lambda}^{\mathrm{AA}}\right)_{\lambda \in(0, \infty)}$ which is incomparable with $T_{L}$. 
In [4], the following results and proposition are obtained.

(1) If $\left(T_{\lambda}^{D}\right)_{\lambda \in(0, \infty)}$ is the Dombi family of $t$-norms and $\left\{x_{n}\right\}_{n \in \mathbb{N}}$ is a sequence of elements from $(0,1]$ such that $\lim _{n \rightarrow \infty} x_{n}=1$, then we have the following equivalence:

$$
\sum_{n=1}^{\infty}\left(1-x_{n}\right)^{\curlywedge}<\infty \Longleftrightarrow \lim _{n \rightarrow \infty}\left(T_{\curlywedge}^{D}\right)_{i=n}^{\infty} x_{i}=1
$$

(2) If $\left(T_{\lambda}^{\mathrm{SW}}\right)_{\lambda \in(-1, \infty]}$ is the Sugeno-Weber family of $t$-norms and $\left\{x_{n}\right\}_{n \in \mathbb{N}}$ is a sequence of elements from $(0,1]$ such that $\lim _{n \rightarrow \infty} x_{n}=1$, then we have the following equivalence:

$$
\sum_{n=1}^{\infty}\left(1-x_{n}\right)<\infty \Longleftrightarrow \lim _{n \rightarrow \infty}\left(T_{\mathcal{\lambda}}^{\mathrm{SW}}\right)_{i=n}^{\infty} x_{i}=1
$$

(3) The equivalence (3.10) holds also for the family $\left(T_{\lambda}^{\mathrm{AA}}\right)_{\lambda \in(0, \infty)}$, that is,

$$
\sum_{n=1}^{\infty}\left(1-x_{n}\right)^{\lambda}<\infty \Longleftrightarrow \lim _{n \rightarrow \infty}\left(T_{\lambda}^{\mathrm{AA}}\right)_{i=n}^{\infty} x_{i}=1
$$

Proposition 3.2. Let $\left\{x_{n}\right\}_{n \in \mathbb{N}}$ be a sequence of numbers from $[0,1]$ such that $\lim _{n \rightarrow \infty} x_{n}=1$ and $T$ a t-norm of H-type. Then $\lim _{n \rightarrow \infty} \mathbf{T}_{i=n}^{\infty} x_{i}=\lim _{n \rightarrow \infty} \mathbf{T}_{i=1}^{\infty} x_{n+i}=1$.

\section{The Main Results}

Let $(X, \mp, T)$ be a Menger PN-space with the $t$-norm $T$ satisfying condition $\sup _{a<1} T(a, a)=1$ and $A$ a nonempty subset of $X$. If $\sup _{t>0} D_{A}(t)=1$, where

$$
D_{A}(t)=\sup _{s<t} \inf _{p, q \in A} F_{p-q}(s), \quad \forall s, t \in \mathbb{R}
$$

then $A$ is called a probabilistically bounded set. Let $\Omega_{X}$ be the collection of all nonempty closed probabilistically bounded subsets of $X$. For any $A, B \in \Omega_{X}$, define the distribution functions $F_{A, B}$ and $F_{A}$ by

$$
\begin{gathered}
F_{A, B}(t)=\sup _{s<t} T\left(\inf _{a \in A} \sup _{b \in B} F_{a-b}(s), \inf _{b \in B} \sup _{a \in A} F_{a-b}(s)\right), \quad \forall s, t \in \mathbb{R}, \\
F_{A}(t)=\sup _{s<t} \sup _{a \in A} F_{a}(s), \quad \forall s, t \in \mathbb{R},
\end{gathered}
$$

respectively. 
Let $\widetilde{S}_{i}, \widetilde{S}_{j}: X \rightarrow W(Y)$ be two fuzzy operators satisfying the following condition (I).

(I) There exist two mappings $a, b: X \rightarrow(0,1]$ such that, for all $x \in X$, the $\operatorname{set}\left(\widetilde{S}_{i x}\right)_{a(x)} \in$ $\Omega_{Y}$ and $\left(\tilde{S}_{j x}\right)_{b(x)} \in \Omega_{Y}$.

We note that

$$
\left(\widetilde{S}_{i x}\right)_{a(x)}=\left\{y \mid \widetilde{S}_{i x}(y) \geq a(x)\right\} \in \Omega_{Y},
$$

where $a(x) \in(0,1)$ is a real number and $\widetilde{S}_{i x} \in W(Y)$ is a fuzzy set in $Y$ decided by the fuzzy operator $\widetilde{S}_{i}$ at $x \in X$. By using each pair of fuzzy operators $\widetilde{S}_{i}$ and $\widetilde{S}_{j}$, we can define two set-valued mappings $S_{i}$ and $S_{j}$ as follows:

$$
\left\{\begin{array}{lll}
S_{i}: X \longrightarrow \Omega_{Y}, & x \longmapsto\left(\widetilde{S}_{i x}\right)_{a(x)^{\prime}} \quad \forall x \in X, \\
S_{j}: X \longrightarrow \Omega_{Y}, & x \longmapsto\left(\widetilde{S}_{j x}\right)_{b(x)^{\prime}} \quad \forall x \in X .
\end{array}\right.
$$

In the sequel, for some $i, j \in \mathbb{N}, S_{i}$ and $S_{j}$ are called the set-valued mappings induced by the fuzzy mappings $\widetilde{P}$ and $\widetilde{S_{j}}$, respectively.

We need the following lemma and definitions.

Lemma 4.1 (see [7]). Let $(X, \mathcal{F}, T)$ be a Menger PN-space with a t-norm $T$ satisfying $\sup _{a<1} T(a, a)=1$ and let $A, B \in \Omega_{X}$. Then we have the following.

(1) $F_{A}(0)=0$.

(2) $F_{A}(t)=1$ for all $t>0$ if and only if $\theta \in A$.

(3) $F_{\alpha A}(t)=F_{A}(t /|\alpha|)$ for all $\alpha \in \mathbb{R}$ with $\alpha \neq 0$.

(4) If $\theta \in B$, then we have $F_{A}(t) \geq F_{A, B}(t)$ for all $t \in \mathbb{R}$.

Definition 4.2. Let $(X, \hat{\mathscr{F}}, T)$ and $(Y, \mathscr{F}, T)$ be two Menger PN-spaces. A set-valued mapping $P: D(P) \subset X \rightarrow \Omega_{Y}$ is said to be $\tau$-closed if, for any $x_{n} \in D(P)$ and $y_{n} \in P\left(x_{n}\right)$, whenever $x_{n} \stackrel{\tau_{X}}{\rightarrow} x$ and $y_{n} \stackrel{\tau_{Y}}{\rightarrow} y$, we have $x \in D(P)$ and $y \in P(x)$.

Definition 4.3. A function $\Psi:[0,1] \rightarrow[0,1]$ is said to satisfy the condition $(\Psi)$ if it is nondecreasing and $\lim _{n \rightarrow \infty} \Psi^{n}(t)=1$ for all $t \in[0,1]$. all $t \in[0,1)$.

It is easy to prove that if $\Psi:[0,1] \rightarrow[0,1]$ satisfies the condition $(\Psi)$, then $\Psi(t)>t$ for

Definition 4.4 (see [7]). Let $(X, \widehat{\Psi}, T)$ and $(Y, \mathcal{F}, T)$ be two Menger PN-spaces and $P: D(P) \subset$ $X \rightarrow 2^{Y}, Q: D(Q) \subset X \rightarrow 2^{Y}$ be two set-valued mappings. Let $\Gamma_{1}, \Gamma_{2}: X \rightarrow L(Y, X)$ be two mappings, where $L(Y, X)$ denotes the space of all linear operators from $Y$ to $X$. $\left(\Gamma_{1}, \Gamma_{2}\right)$ is called a probabilistic $\Psi$-contractor couple of $P$ and $Q$ if there exists a function $\Psi:[0,1] \rightarrow$ $[0,1]$ satisfying the condition $(\Psi)$ such that

$$
F_{P\left(x+\Gamma_{1}(x) y\right), Q(x)+y}(t) \geq \Psi\left(\min \left\{F_{y}(t), F_{Q(x)}(t), F_{P\left(x+\Gamma_{1}(x) y\right)}(t)\right\}\right)
$$


for all $x \in D(Q), y \in\left\{y \in Y: x+\Gamma_{1}(x) y \in D(P)\right\}, t \geq 0$, and

$$
F_{Q\left(x+\Gamma_{2}(x) y\right), P(x)+y}(t) \geq \Psi\left(\min \left\{F_{y}(t), F_{P(x)}(t), F_{Q\left(x+\Gamma_{2}(x) y\right)}(t)\right\}\right)
$$

for all $x \in D(P), y \in\left\{y \in Y: x+\Gamma_{2}(x) y \in D(Q)\right\}$, and $t \geq 0$.

Now, we introduce two algorithms for our main results as follows.

Algorithm 1. Let $(X, \hat{\mathscr{\Psi}}, T)$ be an N.A. Menger PN-space with a $t$-norm $T$ and $\left(Y, \mathcal{F}, T_{Y}\right)$ be a Menger PN-space with a $t$-norm $T_{Y}$. Let $f, g$ be two operators from $X$ into itself, $\left\{\widetilde{S}_{n}\right\}_{n=1}^{\infty}$ a sequence of fuzzy operators from $X$ into $W(X)$ satisfying the condition (I), and $S_{n}$ the $\tau$ closed set-valued operators induced by the fuzzy operators $\widetilde{S}_{n}$ for all $n \in \mathbb{N}$. Let $\Gamma_{1}, \Gamma_{2}: X \rightarrow$ $L(Y, X)$ and $\Psi:[0,1] \rightarrow[0,1]$ satisfy the condition $(\Psi)$. Suppose that

(i) $S_{i}(x) \subset f(X)$ and $S_{j}(x) \subset g(X)$ for all $x \in X$;

(ii) $x+\Gamma_{1}(x) y \in D\left(S_{i}\right)$ for all $x \in D\left(S_{j}\right)$ and $y \in Y, x+\Gamma_{2}(x) y \in D\left(S_{j}\right)$ for all $x \in D\left(S_{i}\right)$ and $y \in Y$;

(iii) $\left(\Gamma_{1}, \Gamma_{2}\right)$ is a probabilistic $\Psi$-contractor couple of $S_{i}$ and $S_{j}$;

(iv) for all $x \in D\left(S_{j}\right)$ and $y \in S_{j}(x)$, there exists $v \in S_{i}\left(x+\Gamma_{1}(x) y\right)$ such that

$$
F_{v}(t) \geq F_{S_{i}\left(x+\Gamma_{1}(x) y\right), S_{j}(x)-y}(t), \quad \forall t \geq 0,
$$

and, for all $x \in D\left(S_{i}\right)$ and $y \in S_{i}(x)$, there exists $w \in S_{j}\left(x+\Gamma_{2}(x) y\right)$ such that

$$
F_{w}(t) \geq F_{S_{j}\left(x+\Gamma_{2}(x) y\right), S_{i}(x)-y}(t), \quad \forall t \geq 0 .
$$

For any $x_{0} \in D\left(S_{j}\right)$ and $y_{0} \in S_{j}\left(x_{0}\right)$, put $x_{1}=\left(1-\alpha_{0}\right) x_{0}+\alpha_{0}\left(x_{0}-\Gamma_{1}\left(x_{0}\right) y_{0}\right)$, where $\alpha_{0} \in(0,1]$ is a real number. It follows from the assumption (ii) that $x_{1} \in D\left(S_{i}\right)$. Replacing $x$ and $y$ by $x_{0}$ and $-y_{0}$ in (4.6), respectively, from (3.11) of Lemma 4.1, the assumption (iii), and $\theta \in S_{j}\left(x_{0}\right)-y_{0}$, it follows that

$$
\begin{aligned}
F_{S_{i}\left(x_{1}\right)}(t) & =F_{S_{i}\left(\left(1-\alpha_{0}\right) x_{0}+\alpha_{0}\left(x_{0}-\Gamma_{1}\left(x_{0}\right) y_{0}\right)\right)}(t) \\
& \geq F_{S_{i}\left(\left(1-\alpha_{0}\right) x_{0}+\alpha_{0}\left(x_{0}-\Gamma_{1}\left(x_{0}\right) y_{0}\right)\right), S_{j}\left(x_{0}\right)-y_{0}}(t) \\
& \geq \Psi\left(\min \left\{F_{y_{0}}(t), F_{S_{j}\left(x_{0}\right)}(t), F_{S_{i}\left(x_{1}\right)}(t)\right\}\right), \quad \forall t \geq 0 .
\end{aligned}
$$

Since $y_{0} \in S_{j}\left(x_{0}\right)$ and $F_{S_{j}\left(x_{0}\right)}(t)=\sup _{s<t} \sup _{a \in S_{j}\left(x_{0}\right)} F_{a}(s)$, it follows that $\sup _{a \in S_{j}\left(x_{0}\right)} F_{a}(s) \geq$ $F_{y_{0}}(s)$ and so

$$
F_{S_{j}\left(x_{0}\right)}(t) \geq \sup _{s<t} F_{y_{0}}(s)=F_{y_{0}}(t), \quad \forall t \geq 0
$$

Since $F_{y_{0}}$ is left continuous, now we have

$$
F_{S_{i}\left(x_{1}\right)}(t) \geq \Psi\left(\min \left\{F_{y_{0}}(t), F_{S_{i}\left(x_{1}\right)}(t)\right\}\right), \quad \forall t \geq 0,
$$


and so $F_{S_{i}\left(x_{1}\right)}(t) \geq F_{y_{0}}(t)$ for all $t>0$. In fact, if there exists $t_{0}>0$ such that $F_{S_{i}\left(x_{1}\right)}\left(t_{0}\right)<F_{y_{0}}\left(t_{0}\right)$, then it follows from (4.12) that

$$
F_{S_{i}\left(x_{1}\right)}\left(t_{0}\right) \geq \Psi\left(F_{S_{i}\left(x_{1}\right)}\left(t_{0}\right)\right)>F_{S_{i}\left(x_{1}\right)}\left(t_{0}\right),
$$

which is a contradiction. Therefore, $F_{S_{i}\left(x_{1}\right)}(t) \geq F_{y_{0}}(t)$ for all $t>0$. Thus, from (4.12), we have

$$
F_{S_{i}\left(x_{1}\right)}(t) \geq F_{S_{i}\left(x_{1}\right), S_{j}\left(x_{0}\right)-y_{0}}(t) \geq \Psi\left(F_{y_{0}}(t)\right), \quad \forall t \geq 0
$$

By the assumption (iv) and (4.14), for any $\theta \in S_{j}\left(x_{0}\right)-y_{0}$, there exists $y_{1} \in S_{i}\left(x_{1}\right)$ such that

$$
F_{y_{1}}(t) \geq F_{S_{i}\left(x_{1}\right), S_{j}\left(x_{0}\right)-y_{0}}(t) \geq \Psi\left(F_{y_{0}}(t)\right), \quad \forall t>0 .
$$

Let $x_{2}=\left(1-\alpha_{1}\right) x_{1}+\alpha_{1}\left(x_{1}-\Gamma_{2}\left(x_{1}\right) y_{1}\right)$, where $\alpha_{1}$ is a real number which satisfies inequality $0<$ $\alpha_{1} \leq \alpha_{0} \leq 1$. By the assumption (ii), we know that $x_{2} \in D\left(S_{j}\right)$. Similarly, since $\theta \in S_{i}\left(x_{1}\right)-y_{1}$, it follows from (4.6) that

$$
\begin{aligned}
F_{S_{j}\left(x_{2}\right)}(t) & =F_{S_{j}\left(\left(1-\alpha_{1}\right) x_{1}+\alpha_{1}\left(x_{1}-\Gamma_{2}\left(x_{1}\right) y_{1}\right)\right.}(t) \\
& \geq F_{S_{j}\left(\left(1-\alpha_{1}\right) x_{1}+\alpha_{1}\left(x_{1}-\Gamma_{2}\left(x_{1}\right) y_{1}\right)\right), S_{i}\left(x_{1}\right)-y_{1}}(t) \\
& \geq \Psi\left(\min \left\{F_{y_{1}}(t), F_{S_{i}\left(x_{1}\right)}(t), F_{S_{j}\left(x_{2}\right)}(t)\right\}\right) \\
& \geq \Psi\left(\min \left\{F_{y_{1}}(t), F_{S_{j}\left(x_{2}\right)}(t)\right\}\right), \quad \forall t \geq 0 .
\end{aligned}
$$

It is easy to check that $F_{y_{1}}(t) \leq F_{S_{j}\left(x_{2}\right)}(t)$ for all $t>0$ and so it follows from (4.15) that

$$
F_{S_{j}\left(x_{2}\right)}(t) \geq F_{S_{j}\left(x_{2}\right), S_{i}\left(x_{1}\right)-y_{1}}(t) \geq \Psi\left(F_{y_{1}}(t)\right) \geq \Psi^{2}\left(F_{y_{0}}(t)\right), \quad \forall t>0
$$

Now, for any $\theta \in S_{i}\left(x_{1}\right)-y_{1}$, the assumption (iv) implies that there exists $y_{2} \in S_{j}\left(x_{2}\right)$ such that

$$
F_{y_{2}}(t) \geq F_{S_{j}\left(x_{2}\right), S_{i}\left(x_{1}\right)-y_{1}}(t) \geq \Psi^{2}\left(F_{y_{0}}(t)\right), \quad \forall t>0 .
$$

Inductively, we can get two sequences $\left\{x_{n}\right\}$ in $X$ and $\left\{y_{n}\right\}$ in $Y$, respectively, as follows:

$$
\left\{\begin{array}{l}
x_{2 n+1}=\left(1-\alpha_{2 n}\right) x_{2 n}+\alpha_{2 n}\left(x_{2 n}-\Gamma_{1}\left(x_{2 n}\right) y_{2 n}\right), \\
x_{2 n+2}=\left(1-\alpha_{2 n+1}\right) x_{2 n+1}+\alpha_{2 n+1}\left(x_{2 n+1}-\Gamma_{2}\left(x_{2 n+1}\right) y_{2 n+1}\right), \quad \forall n \geq 0,
\end{array}\right.
$$

where $\left\{\alpha_{n}\right\}$ is a real monotone decreasing sequence in $(0,1]$ and $\alpha_{n} \rightarrow \alpha \in(0,1]$ as $n \rightarrow \infty$; the sequence $\left\{y_{n}\right\}$ in $Y$ is defined by (2.13) and satisfies the following:

$$
F_{y_{n}}(t) \geq \Psi^{n}\left(F_{y_{0}}(t)\right), \quad \forall t>0
$$


Algorithm 2. Let $(X, \widehat{\mathscr{\Psi}}, T)$ be a N.A. Menger PN-space with a $t$-norm $T,\left(Y, \mathcal{F}, T_{Y}\right)$ a Menger PN-space with a $t$-norm $T_{Y}$, and $A, V: X \rightarrow X$ be two operators. Let $\left\{\widetilde{S}_{n}\right\}_{n=1}^{\infty}$ a sequence of fuzzy operators from $X$ into $W(X)$ satisfying the condition (I) and $S_{n}$ the set-valued operators induced by the fuzzy operators $\widetilde{S}_{n}$ for all $n \geq 1$. Let $\Gamma_{1}, \Gamma_{2}: X \rightarrow L(Y, X)$ and $\Psi:[0,1] \rightarrow[0,1]$ satisfy the condition $(\Psi)$. Suppose that the conditions (ii)-(iv) in Algorithm 1 are satisfied. If

(i)' $S_{i}(x) \subset X-A(X)$ and $S_{j}(x) \subset X-V(X)$ for all $x \in X$, then, for any $x_{0} \in D\left(S_{j}\right)$ and $y_{0} \in S_{j}\left(x_{0}\right)$, we have two sequences $\left\{x_{n}\right\}$ in $X$ and $\left\{y_{n}\right\}$ in $Y$, respectively, defined as follows:

$$
\left\{\begin{array}{l}
x_{2 n+1}=x_{2 n}-\Gamma_{1}\left(x_{2 n}\right) y_{2 n}, \\
x_{2 n+2}=x_{2 n+1}-\Gamma_{2}\left(x_{2 n+1}\right) y_{2 n+1}, \quad \forall n \geq 0
\end{array}\right.
$$

where the sequence $\left\{y_{n}\right\}$ in $Y$ is defined by (2.13).

Now, we state our main results by using the similar ideas as in [9].

Theorem 4.5. Let $(X, \hat{\mathcal{F}}, T)$ be an N.A. Menger PN-space with a t-norm $T$ and $\left(Y, \mathcal{F}, T_{Y}\right)$ be a Menger PN-space with a t-norm $T_{Y}$. Let $f, g,\left\{\widetilde{S}_{n}\right\}_{n=1}^{\infty}, S_{n}, \Gamma_{1}, \Gamma_{2}$, and $\Psi$ be the same as in Algorithm 1. Suppose that the conditions (i)-(iv) in Algorithm 1 hold and the following conditions are satisfied:

(v) $g(X)$ is $x_{0}$-joint orbitally complete for some $x_{0} \in X$;

(vi) there exists a constant $M>0$ such that, for any constant $\lambda_{1}>\lambda_{2}>0$,

$$
\begin{cases}\widehat{F}_{\lambda_{1} \Gamma_{1}(x) y}(t) \geq F_{y}\left(\frac{t}{\lambda_{2} M}\right), \quad \forall x \in D\left(S_{j}\right), y \in Y, t \geq 0 \\ \widehat{F}_{\lambda_{1} \Gamma_{2}(x) y}(t) \geq F_{y}\left(\frac{t}{\lambda_{2} M}\right), \quad \forall x \in D\left(S_{i}\right), y \in Y, t \geq 0\end{cases}
$$

(vii) there exist $x_{0} \in D\left(S_{j}\right)$ and $y_{0} \in S_{j}\left(x_{0}\right)$ such that the $t$-norm $T$ satisfies the following condition:

$$
\lim _{n \rightarrow \infty} \mathbf{T}_{i=n}^{\infty} \Psi^{i}\left(F_{y_{0}}\left(\frac{t}{\lambda M}\right)\right)=1, \quad \forall t \geq 0
$$

where $\lambda$ is a real nonnegative number.

Then the following system of nonlinear operator equations:

$$
\left\{\begin{array}{l}
\theta=f(x) \\
\theta=g(x)
\end{array}\right.
$$

has a solution $z$ such that $\{f(z)\}=\{g(z)\} \subset \bigcap_{i=1}^{\infty} S_{i}(z)$. Further, $\left\{x_{n}\right\}$-converges to a solution of (4.24) and $\left\{y_{n}\right\} \tau$-converges to $\theta$, where $\left\{x_{n}\right\}$ in $X$ and $\left\{y_{n}\right\}$ in $Y$ are two sequences generated by Algorithm 1. 
Proof. By (4.19), (4.20), and the assumption (vi), since $\left\{\alpha_{n}\right\} \subset(0,1]$ is a monotone decreasing sequence with $\alpha_{n} \rightarrow \alpha \in(0,1]$, we have

$$
\left\{\begin{array}{l}
\widehat{F}_{x_{2 n+1}-x_{2 n}}(t)=\widehat{F}_{\alpha_{2 n} \Gamma_{1}\left(x_{2 n}\right) y_{2 n}}(t) \geq F_{y_{2 n}}\left(\frac{t}{\alpha M}\right) \geq \Psi^{2 n}\left(F_{y_{0}}\left(\frac{t}{\alpha M}\right)\right), \\
\widehat{F}_{x_{2 n+2}-x_{2 n+1}}(t)=\widehat{F}_{\alpha_{2 n+1} \Gamma_{2}\left(x_{2 n+1}\right) y_{2 n+1}}(t) \geq F_{y_{2 n+1}}\left(\frac{t}{\alpha M}\right) \geq \Psi^{2 n+1}\left(F_{y_{0}}\left(\frac{t}{\alpha M}\right)\right), \quad \forall t \geq 0,
\end{array}\right.
$$

which imply that

$$
\widehat{F}_{x_{n+1}-x_{n}}(t) \geq \Psi^{n}\left(F_{y_{0}}\left(\frac{t}{\alpha M}\right)\right), \quad \forall t>0, n \geq 1
$$

Since $(X, \hat{\mp}, T)$ is N.A. Menger PN-space, it follows from (4.26) that, for any positive integers $m, n(m>n)$,

$$
\begin{aligned}
& \widehat{F}_{x_{n}-x_{m}}(t) \\
& \geq T\left(\widehat{F}_{x_{n}-x_{n+1}}(t), \widehat{F}_{x_{n+1}-x_{m}}(t)\right) \\
& \geq T\left(\widehat{F}_{x_{n}-x_{n+1}}(t), T\left(\widehat{F}_{x_{n+1}-x_{n+2}}(t), \widehat{F}_{x_{n+2}-x_{m}}(t)\right)\right) \\
& \geq T\left(\widehat{F}_{x_{n}-x_{n+1}}(t), T(\widehat{F}_{x_{n+1}-x_{n+2}}(t), \underbrace{T\left(\cdots, T\left(\widehat{F}_{x_{m-2}-x_{m-1}}(t), \widehat{F}_{x_{m-1}-x_{m}}(t)\right) \cdots\right)}_{(m-n)-3})\right) \\
& \geq T\left(\Psi^{n}\left(F_{y_{0}}\left(\frac{t}{\alpha M}\right)\right), T\left(\Psi^{n+1}\left(F_{y_{0}}\left(\frac{t}{\alpha M}\right)\right),\right.\right. \\
& \underbrace{T\left(\cdots, T\left(\Psi^{m-2}\left(F_{y_{0}}\left(\frac{t}{\alpha M}\right)\right), \Psi^{m-1}\left(F_{y_{0}}\left(\frac{t}{\alpha M}\right)\right)\right) \cdots\right)}_{(m-n)-3})) \\
& =\mathbf{T}_{i=n}^{m-1} \Psi^{i}\left(F_{y_{0}}\left(\frac{t}{\alpha M}\right)\right) \\
& \geq \mathbf{T}_{i=n}^{\infty} \Psi^{i}\left(F_{y_{0}}\left(\frac{t}{\alpha M}\right)\right), \quad \forall t \geq 0 .
\end{aligned}
$$


Since $\lim _{n \rightarrow \infty} \mathbf{T}_{i=n}^{\infty} \Psi^{i}\left(F_{y_{0}}(t / \alpha M)\right)=1$, it follows that, for all $\lambda \in(0,1)$ and $t>0$, there exists a positive integer $n(t, \lambda)$ such that, for all $n \geq n(t, \lambda)$ and $m>n$,

$$
\mathbf{T}_{i=n}^{\infty} \Psi^{i}\left(F_{y_{0}}\left(\frac{t}{\alpha M}\right)\right)>1-\lambda
$$

and so

$$
\widehat{F}_{x_{n}-x_{m}}(t) \geq \mathbf{T}_{i=n}^{m-1} \Psi^{i}\left(F_{y_{0}}\left(\frac{t}{\alpha M}\right)\right) \geq \mathbf{T}_{i=n}^{\infty} \Psi^{i}\left(F_{y_{0}}\left(\frac{t}{\alpha M}\right)\right)>1-\lambda
$$

Hence $\left\{x_{n}\right\}$ is a $\tau$-Cauchy sequence in $X$. Since $g(X)$ is $x_{0}$-joint orbitally complete, we can assume that $x_{n} \stackrel{\tau_{X}}{\rightarrow} z \in X$. Moreover, by (4.20), it is easy to see that $\lim _{n \rightarrow \infty} F_{y_{n}}(t)=1$ for all $t>0$ and so $y_{n} \stackrel{\tau_{x}}{\rightarrow} \theta$. Since $S_{i}$ and $S_{j}$ are $\tau$-closed, it follows from (4.19) and the assumption (i) that

$$
\left\{\begin{array}{l}
\theta \in S_{i}(z), \\
\theta \in S_{j}(z), \quad \forall i, j \geq 1
\end{array}\right.
$$

that is, $z$ is a solution of (4.24) and $\{f(z)\}=\{g(z)\} \subset \bigcap_{i=1}^{\infty} S_{i}(z)$. This completes the proof.

From Theorem 4.5, we have the following.

Corollary 4.6. Let $(X, \hat{\mathscr{F}}, T)$ be an N.A. Menger PN-space with a t-norm $T$ and $\left(Y, \mathcal{F}, T_{Y}\right)$ a Menger $P N$-space with a $t$-norm $T_{Y}$. Let $f, g$ be two operators from $X$ into itself, $\left\{\widetilde{S}_{n}\right\}_{n=1}^{\infty}$ a sequence of fuzzy operators from $X$ into $W(X)$ satisfying the condition (I), and $S_{n}$ the $\tau$-closed set-valued operators induced by the fuzzy operators $\widetilde{S}_{n}$ for all $n \geq 1$. Let $\Gamma_{1}, \Gamma_{2}: X \rightarrow L(Y, X)$ and $\Psi:[0,1] \rightarrow$ $[0,1]$ satisfy the condition ( $\Psi)$. Suppose that the conditions (i)-(iv) in Algorithm 1 and (v)-(vi) in Theorem 4.5 are satisfied. If $t$-norm $T$ is of H-type, then the conclusions of Theorem 4.5 still hold.

Proof. By Proposition 3.2, we know that all the conditions of Theorem 4.5 are satisfied. Thus the conclusions of Theorem 4.5 still hold.

Corollary 4.7. Let $\left(X, \hat{\mathcal{F}},\left(T_{\lambda}^{D}\right)\right)$ for some $\lambda>0$ be a N.A. Menger PN-space and $\left(Y, \mathcal{F}, T_{Y}\right)$ be a Menger PN-space. Let $f, g$ be two operators from $X$ into itself, $\left\{\widetilde{S}_{n}\right\}_{n=1}^{\infty}$ be a sequence of fuzzy operators from X into $W(X)$ satisfying the condition (I) and $S_{n}$ be the $\tau$-closed set-valued operators induced by the fuzzy operators $\widetilde{S}_{n}$ for all $n \geq 1$. Let $\Gamma_{1}, \Gamma_{2}: X \rightarrow L(Y, X)$ and $\Psi:[0,1] \rightarrow$ $[0,1]$ satisfy the condition $(\Psi)$. Suppose that the conditions (i)-(iv) in Algorithm 1 and (v)-(vi) of Theorem 4.5 are satisfied. If there exist $x_{0} \in D\left(S_{j}\right)$ and $y_{0} \in S_{j}\left(x_{0}\right)$ for some $j \in \mathbb{N}$ such that $\sum_{n=1}^{\infty}\left(1-\Psi^{n}\left(F_{y_{0}}(t / \mu M)\right)\right)^{\lambda}<\infty$ for all $t \geq 0$, where $\mu>0$ is a constant, then the conclusions of Theorem 4.5 still hold. 
Proof. From the equivalence (3.10), we have

$$
\sum_{n=1}^{\infty}\left(1-\Psi^{n}\left(F_{y_{0}}\left(\frac{t}{\mu M}\right)\right)\right)^{\curlywedge}<\infty \Longleftrightarrow \lim _{n \rightarrow \infty}\left(T_{\curlywedge}^{D}\right)_{i=n}^{\infty} \Psi^{i}\left(F_{y_{0}}\left(\frac{t}{\mu M}\right)\right)=1
$$

Corollary 4.8. Let $\left(X, \widehat{\mathcal{F}},\left(T_{\lambda}^{S W}\right)\right)$ for some $\lambda>-1$ be an N.A. Menger PN-space. Let $\left(Y, \mathcal{F}, T_{Y}\right), f$, $g,\left\{\widetilde{S}_{n}\right\}_{n=1}^{\infty}, S_{n}, \Gamma_{1}, \Gamma_{2}$, and $\Psi$ be the same as in Theorem 4.5. Suppose that the conditions (i)-(iv) in Algorithm 1 and (v)-(vi) in Theorem 4.5 are satisfied. If there exist $x_{0} \in D\left(S_{j}\right)$ and $y_{0} \in S_{j}\left(x_{0}\right)$ for some $j \in \mathbb{N}$ such that $\sum_{n=1}^{\infty}\left(1-\Psi^{n}\left(F_{y_{0}}(t / \mu M)\right)\right)<\infty$ for all $t \geq 0$, where $\mu>0$ is a constant, then the conclusions of Theorem 4.5 still hold.

Proof. From the equivalence (3.11), we have

$$
\sum_{n=1}^{\infty}\left(1-\Psi^{n}\left(F_{y_{0}}\left(\frac{t}{\mu M}\right)\right)\right)<\infty \Longleftrightarrow \lim _{n \rightarrow \infty}\left(T_{\mathcal{\lambda}}^{S W}\right)_{i=n}^{\infty} \Psi^{i}\left(F_{y_{0}}\left(\frac{t}{\mu M}\right)\right)=1
$$

Remark 4.9. Since

$$
T_{L} \in \bigcup_{\lambda \in(-1, \infty)} T_{\lambda}^{S W}
$$

it is easy to see that Corollary 4.8 is a generalization of the corresponding result in Fang [9].

Corollary 4.10. Let $\left(X, \hat{\bar{F}},\left(T_{\lambda}^{\mathrm{AA}}\right)\right)$ for some $\lambda>0$ be an N.A. Menger PN-space with a t-norm $T$. Let $\left(Y, \mathcal{F}, T_{Y}\right), f, g,\left\{\widetilde{S}_{n}\right\}_{n=1}^{\infty}, S_{n}, \Gamma_{1}, \Gamma_{2}$, and $\Psi$ be the same as in Theorem 4.5. Suppose that the conditions (i)-(iv) in Algorithm 1 and (v)-(vi) of Theorem 4.5 are satisfied. If there exist $x_{0} \in D\left(S_{j}\right)$ and $y_{0} \in S_{j}\left(x_{0}\right)$ for some $j \geq 1$ such that $\sum_{n=1}^{\infty}\left(1-\Psi^{n}\left(F_{y_{0}}(t / \mu M)\right)\right)^{\lambda}<\infty$ for all $t \geq 0$, where $\mu>0$ is a constant, then the conclusions of Theorem 4.5 still hold.

Proof. From the equivalence (3.12), we have

$$
\sum_{n=1}^{\infty}\left(1-\Psi^{n}\left(F_{y_{0}}\left(\frac{t}{\mu M}\right)\right)\right)^{\lambda}<\infty \Longleftrightarrow \lim _{n \rightarrow \infty}\left(T_{\lambda}^{\mathrm{AA}}\right)_{i=n}^{\infty} \Psi^{i}\left(F_{y_{0}}\left(\frac{t}{\mu M}\right)\right)=1 .
$$

Corollary 4.11. Let $(X, \hat{\mp}, T)$ be an N.A. Menger PN-space and $L: X \rightarrow \Omega_{X}$ satisfy the following condition:

$$
F_{L x, L y}(t) \geq \Psi\left(\min \left\{F_{x-y}(t), F_{x-L(x)}(t), F_{y-L(y)}(t)\right\}\right), \quad \forall t \geq 0, x, y \in X,
$$

where a mapping $\Psi:[0,1] \rightarrow[0,1]$ satisfies the condition $(\Psi)$. Suppose that the conditions $(i)$ in Algorithm 1 and $(v)$ in Theorem 4.5 are satisfied and there exists $x_{0} \in X$ and $y_{0} \in x_{0}-L\left(x_{0}\right)$ such that $t$-norm $T$ satisfies the following condition:

$$
\lim _{n \rightarrow \infty} \mathbf{T}_{i=n}^{\infty} \Psi^{i}\left(F_{y_{0}}(t)\right)=1, \quad \forall t \geq 0,
$$


and, for all $x \in X$ and $y \in x-L(x)$, there exists $v \in x+y-L(x+y)$ such that

$$
F_{v}(t) \geq F_{x+y-L(x+y), x-L(x)-y}(t), \quad \forall t \geq 0 .
$$

Then there exists a point $x^{*} \in X$ such that $x^{*} \in L x^{*}$, that is, $x^{*}$ is a fixed point of $L$.

Proof. Putting $S_{i}(x)=S_{j}(x)=x-L(x)$ for any fixed $i, j \geq 1$ and $\Gamma_{1}(x)=\Gamma_{2}(x)=I_{X}$, the mappings $S_{i}, S_{j}, \Gamma_{1}$ and $\Gamma_{2}$ satisfy all the hypotheses of Theorem 4.5. Therefore, there exists a point $x^{*} \in X$ such that $\theta \in S_{i}\left(x^{*}\right)=S_{j}\left(x^{*}\right)=x^{*}-L\left(x^{*}\right)$, which means that $x^{*}$ is a fixed point of $T$. This completes the proof.

Theorem 4.12. Let $(X, \hat{\mathscr{F}}, T)$ be an N.A. Menger PN-space with a t-norm $T$ and $\left(Y, \mathcal{F}, T_{Y}\right)$ be a Menger PN-space with a t-norm $T_{Y}$. Let $A, V,\left\{\widetilde{S}_{n}\right\}_{n=1}^{\infty}, S_{n}, \Gamma_{1}, \Gamma_{2}$, and $\Psi$ be the same as in Algorithm 2. Suppose that the conditions (ii)-(iv) in Algorithm 1 and (i)' in Algorithm 2 are satisfied. If

$(\mathrm{v})^{\prime} X-V(X)$ is $x_{0}$-joint orbitally complete for some $x_{0} \in X$,

(vi)' there exists a constant $M>0$ such that

$$
\begin{cases}\widehat{F}_{\Gamma_{1}(x) y}(t) \geq F_{y}\left(\frac{t}{M}\right), & \forall x \in D\left(S_{j}\right), y \in Y, t \geq 0 \\ \widehat{F}_{\Gamma_{2}(x) y}(t) \geq F_{y}\left(\frac{t}{M}\right), & \forall x \in D\left(S_{i}\right), y \in Y, t \geq 0\end{cases}
$$

(vii)' there exist $x_{0} \in D\left(S_{j}\right)$ and $y_{0} \in S_{j}\left(x_{0}\right)$ such that the t-norm $T$ satisfies the following condition:

$$
\lim _{n \rightarrow \infty} \mathbf{T}_{i=n}^{\infty} \Psi^{i}\left(F_{y_{0}}\left(\frac{t}{M}\right)\right)=1, \quad \forall t \geq 0,
$$

then the following system of nonlinear operator equations:

$$
\left\{\begin{array}{l}
x=A(x), \\
x=V(x)
\end{array}\right.
$$

has a solution $z$ such that $\{z-A(z)\}=\{z-V(z)\} \subset \bigcap_{i=1}^{\infty} S_{i}(z)$. Further, $\left\{x_{n}\right\} \tau$-converges to a solution of (4.40) and $\left\{y_{n}\right\} \tau$-converges to $\theta$, where the sequences $\left\{x_{n}\right\}$ in $X$ and $\left\{y_{n}\right\}$ in $Y$ are defined by Algorithm 2.

Proof. Let $f(x)=x-A(x)$ and $g(x)=x-V(x)$ for all $x \in X$. It is obvious that all the conditions of Theorem 4.5 are satisfied. Therefore, the conclusion of Theorem 4.12 follows from Theorem 4.5 immediately.

Remark 4.13. Similarly, we can obtain the conclusions of Theorem 4.12 if we replace the condition (iii) in Theorem 4.12 by the corresponding condition in Proposition 3.2 and the equivalences (3.10)-(3.12), respectively. 


\section{Acknowledgments}

This work was supported by the Korea Research Foundation Grant funded by the Korean Government (KRF-2008-313-C00050), the Sichuan Youth Science and Technology Foundation (08ZQ026-008), and the Open Foundation of Artificial Intelligence of Key Laboratory of Sichuan Province (2009RZ001).

\section{References}

[1] M. Altman, Contractors and Contractor Directions. Theory and Applications, Marcel Dekker, New York, NY, USA, 1977.

[2] M. Altman, "Contractors and fixed points," in Topological Methods in Nonlinear Functional Analysis, vol. 21 of Contemporary Mathematics, pp. 1-14, American Mathematical Society, Providence, RI, USA, 1983.

[3] S. S. Chang, "Probabilistic contractor and the solutions for lonlinear equations in PN-spaces," Chinese Science Bulletin, vol. 35, pp. 1451-1454, 1990.

[4] O. Hadžić and E. Pap, Fixed Point Theory in Probabilistic Metric Spaces, vol. 536 of Theory in Probabilistic Metric Spaces, Kluwer Academic Publishers, Dordrecht, The Netherlands, 2001.

[5] O. Hadžić, E. Pap, and M. Budinčević, "Countable extension of triangular norms and their applications to the fixed point theory in probabilistic metric spaces," Kybernetika, vol. 38, no. 3, pp. 363-381, 2002.

[6] E. P. Klement, R. Mesiar, and E. Pap, Triangular Norms, vol. 8 of Trends in Logic-Studia Logica Library, Kluwer Academic Publishers, Dordrecht, The Netherlands, 2000.

[7] S.-S. Chang, Y. J. Cho, and S. M. Kang, Nonlinear Operator Theory in Probabilistic Metric Spaces, Nova Science, Huntington, NY, USA, 2001.

[8] T. Žikić, Existence of fixed point in fuzzy structures, Ph.D. thesis, Faculty of Science and Mathematics, University of Novi Sad, Vojvodina, Serbia, 2002.

[9] J.-X. Fang, "On nonlinear equations for fuzzy mappings in probabilistic normed spaces," Fuzzy Sets and Systems, vol. 131, no. 3, pp. 357-364, 2002.

[10] Y. J. Cho, N. J. Huang, and S. M. Kang, "Nonlinear equations for fuzzy mappings in probabilistic normed spaces," Fuzzy Sets and Systems, vol. 110, no. 1, pp. 115-122, 2000.

[11] N.-J. Huang, "Completely generalized strongly nonlinear quasi-complementarity problems for fuzzy mappings," Indian Journal of Pure and Applied Mathematics, vol. 28, no. 1, pp. 23-32, 1997.

[12] N.-J. Huang, "Random generalized nonlinear variational inclusions for random fuzzy mappings," Fuzzy Sets and Systems, vol. 105, no. 3, pp. 437-444, 1999.

[13] N.-J. Huang and H.-Y. Lan, "A couple of nonlinear equations with fuzzy mappings in fuzzy normed spaces," Fuzzy Sets and Systems, vol. 152, no. 2, pp. 209-222, 2005.

[14] B. S. Lee and S. J. Cho, "A fixed point theorem for contractive-type fuzzy mappings," Fuzzy Sets and Systems, vol. 61, no. 3, pp. 309-312, 1994.

[15] B. K. Sharma, D. R. Sahu, and M. Bounias, "Common fixed point theorems for a mixed family of fuzzy and crisp mappings," Fuzzy Sets and Systems, vol. 125, no. 2, pp. 261-268, 2002.

[16] O. Hadžić and E. Pap, "New classes of probabilistic contractions and applications to random operators," in Fixed Point Theory and Applications, Y. J. Cho, J. K. Kim, and S. M. Kang, Eds., vol. 4, pp. 97-119, Nova Science, Hauppauge, NY, USA, 2003.

[17] M. Bounias, "A theorem proving the irreversibility of the biological arrow of time based on fixed points in the brain as a compact and $\Delta$-complete topological space," in Computing Anticipatory Systems, vol. 517 of AIP Conference Proceedings, pp. 233-243, American Institute of Physics, 2000.

[18] B. Schweizer and A. Sklar, Probabilistic Metric Spaces, North-Holland Series in Probability and Applied Mathematics, North-Holland, New York, NY, USA, 1983.

[19] O. Hadžić, "A fixed point theorem in Menger spaces," Publications de l'Institut Mathématique, vol. 26, pp. 107-112, 1979. 